\title{
Pediatric Secondary Transfer Percentages: A Retrospective Observational Study
}

Yves Leroux ${ }^{1}$, Jolene Cook ${ }^{1}$, Judah Goldstein ${ }^{1}$, Steve Doucette ${ }^{2}$, Corinne DeMone ${ }^{3}$, Alix Carter ${ }^{4}$, Katrina F. Hurley 5

1. Emergency Medicine, Dalhousie University, Halifax, CAN 2. Research Methods Unit, Nova Scotia Health Authority, Halifax, CAN 3. Emergency Medicine, Nova Scotia Health Authority, Halifax, CAN 4. Division of Emergency Medical Services, Dalhousie University, Halifax, CAN 5. Emergency Department, IWK Health Centre, Halifax, CAN

Corresponding author: Yves Leroux, yleroux@dal.ca

\begin{abstract}
Introduction: Certain adult conditions treated by paramedics, such as myocardial infarction or stroke, have better outcomes if transported to a specialty centre, bypassing local generalist facilities when necessary. Little evidence exists to inform guidelines to identify pediatric patients who would benefit from direct transport to a pediatric centre. This study describes the characteristics of children brought to community emergency departments (ED) who subsequently required transfer to pediatric specialty care.
\end{abstract}

Methods: A retrospective observational cohort study was performed in a metropolitan area with one tertiary pediatric specialty centre and four community EDs. The patient care record database was queried for patients under 16 years old transported by paramedics to a community ED during a five-year period. Secondary transfer to the pediatric specialty centre within 24 hours was identified. The primary outcome was percentage of transfers to specialty care. Descriptive statistics were used to characterize the whole group as well as stratified by age category, chief complaint and Canadian Triage Acuity Scale (CTAS).

Results: A total of 872 pediatric patients were transported to community EDs with 95 (10.9\%) requiring secondary transfer to the pediatric specialty centre. CTAS 1 and 2 were associated with increased secondary transfer $(\mathrm{p}<0.001)$. There were also differences in transfer proportion by chief complaint. There was no association between age or gender and transfer to pediatric specialty care.

Conclusions: This retrospective study shows an association between acuity and certain chief complaints and percentage of secondary transfer to pediatric specialty care.

Categories: Emergency Medicine, Pediatrics

Keywords: pediatrics, transportation of patients, patient transfer, emergency medical services

Received 07/18/2019

Review began 07/22/2019

Review ended 01/21/2020

Published 01/24/2020

๑) Copyright 2020

Leroux et al. This is an open access

article distributed under the terms of the Creative Commons Attribution License CC-BY 3.0., which permits unrestricted use, distribution, and reproduction in any medium, provided the original author and source are credited.

\section{Introduction}

Regionalized systems of healthcare are used to improve the outcomes of patient populations requiring specialized treatment. The integration of prehospital care providers relies on clinical guidelines that allow these patients to be identified appropriately in the field and transported directly to the required resource, bypassing other destinations. In adults, guidelines that enabled paramedics to identify patients with STelevation myocardial infarction, notify the cardiology team and transport the patient directly to the regional specialty centre led to a decreased mortality from $8.9 \%$ to $1.9 \%$ compared to transporting to the nearest hospital [1].

Specialized pediatric centres currently exist throughout North America. Other than traumatic injury, there is a paucity of evidence to inform clinical guidelines that would assist prehospital identification of those patients who would benefit most from direct transport to the pediatric centre instead of the nearest hospital. This knowledge gap has recently been identified as a priority study objective for pediatric prehospital research [2]. Children brought by paramedics to a community emergency department (ED) and subsequently transferred to a tertiary care pediatric hospital may benefit from direct transport from the scene to a pediatric specialty centre.

\section{Study objective}

Our objective was to describe the clinical characteristics of children brought to community EDs who subsequently required transfer to pediatric specialty care.

\section{Materials And Methods Study design and setting}

This retrospective observational study was performed in a metropolitan area served by one tertiary care 


\section{Cureus}

pediatric hospital which sees patients aged 0-15 years old and four community EDs serving all age groups. Prehospital care and transport is performed by a single Advanced Life Support province-wide Emergency Medical Services (EMS) system. This sample consisted of all patients less than 16 years of age transported by paramedics to any of the four community EDs between January 1, 2010 and December 31, 2015.

\section{Data search}

The EMS system maintains a database of dispatch records and electronic patient care records for every request for ambulance service in the province. The search process identified all patients who met the eligibility criteria. Deterministic linkage using first name, last name, sex, date of birth, health card number, date of service and facility address was used to search for subsequent interfacility transfer within 24 hours.

\section{Outcome measures}

The primary outcome was the proportion of patients requiring subsequent transfer to the pediatric specialty centre within 24 hours of being transported from the field to a community hospital by paramedics (secondary transfer). Secondary outcomes were the proportion of transfers by age, chief complaint and Canadian Triage and Acuity Scale (CTAS).

\section{Data analysis}

Baseline demographics were summarized as means and standard deviation for continuous data. Categorical data were summarized as proportions with percentages. Chief complaints were grouped into categories. Secondary transfer proportions in each chief complaint category were shown as percentages. The effect of age, gender, and CTAS category on transfer to the specialty centre were shown as odds ratios (OR) with $95 \%$ confidence intervals (CI). P-values less than 0.05 were considered statistically significant. All analyses were performed using SAS, version 9.4 (SAS Institute, Cary, NC).

\section{Ethics}

This study was approved by the Nova Scotia Health Authority Research Ethics Board (File No. 1021022).

\section{Results}

During the five-year study period, 872 pediatric patients (mean age 9.7 years \pm 4.90 ) were transported by paramedics to a community ED. Of these, 95 (10.9\%) patients were subsequently transferred to the pediatric specialty centre (Table 1 ).

\begin{tabular}{|c|c|c|c|}
\hline Characteristic & No Secondary Transfer ( $n=777,89.1 \%)$ & Secondary Transfers ( $\mathrm{n}=95 ; 10.9 \%)$ & All Patients $(n=872)$ \\
\hline Age, mean $(95 \%$ Cl) & 9.7 years $(0.1-19.3)$ & 9.8 years $(1.1-18.4)$ & 9.7 years $(0.1-19.3)$ \\
\hline Male $(n, \%)$ & $406(52.2 \%)$ & $55(57.9 \%)$ & $461(52.8 \%)$ \\
\hline \multicolumn{4}{|l|}{ CTAS (n, \%) } \\
\hline CTAS 1 & $2(0.3 \%)$ & $7(7.4 \%)$ & $9(1.0 \%)$ \\
\hline CTAS 2 & $92(11.8 \%)$ & $33(34.7 \%)$ & $125(14.3 \%)$ \\
\hline CTAS 3 & $377(48.5 \%)$ & $38(40.0 \%)$ & $415(47.6 \%)$ \\
\hline CTAS 4 & $231(29.7 \%)$ & $14(14.7 \%)$ & $245(28.1 \%)$ \\
\hline CTAS 5 & $65(8.4 \%)$ & $2(2.1 \%)$ & $67(7.7 \%)$ \\
\hline
\end{tabular}

\section{TABLE 1: Demographic data of the study population}

Cl, confidence interval; CTAS, Canadian Triage Acuity Scale

A total of 461 (52.8\%) patients were male with no significant difference in odds of transport $(\mathrm{p}=0.48)$. Nearly half of all patients were triaged as CTAS $3(n=415 ; 47.6 \%)$. There was a clear trend in increasing odds of transfer as CTAS decreased, with a statistically significant odds ratio for CTAS 2 (OR: 11.6, 95\% CI: 2.6850.3, $\mathrm{p}=0.001$ ) and CTAS 1 (OR: 113.4, 95\% CI: 13.6-941.2, $\mathrm{p}<0.0001$ ) relative to CTAS 5 (Table 2). 


\section{Cureus}

\begin{tabular}{|l|l|l|}
\hline CTAS & OR (95\% C CI) & P-Value \\
\hline 1 & & $<0.0001$ \\
\hline 2 & $113.36(13.64,941.8)$ & 0.001 \\
\hline 3 & $11.61(2.68,50.33)$ & 0.11 \\
\hline 4 & $3.28(0.77,13.94)$ & 0.38 \\
\hline 5 & $1.97(0.44,8.92)$ & Reference \\
\hline Male vs. female & Reference & 0.48 \\
\hline Age (per year) & $1.18(0.75,1.86)$ & 0.9 \\
\hline
\end{tabular}

TABLE 2: ORs of transfer by CTAS, sex and years of age

OR, odds ratio; Cl, confidence interval; CTAS, Canadian Triage Acuity Scale

There was no significant trend in transfer by age $(\mathrm{p}=0.9)$. There were only four patients younger than one year brought to a community ED, none of whom were subsequently transferred.

The 36 chief complaints in the sample were grouped into 16 categories for analysis. The most common complaint was "minor trauma” ( $\mathrm{n}=406)$. Categories of "major trauma”, “diabetic complaint”, "altered mental status" and "overdose/poisoning” had the highest percentage of secondary transfer at 50\%, 50\%, $41.7 \%$ and $24.4 \%$, respectively (Table 3 ).

\begin{tabular}{|c|c|c|c|c|c|}
\hline Chief Complaint & Overall & Transferred (n=95) & Not Transferred (n=777) & Proportion Transferred & P-Value \\
\hline Pain/nausea & $67(7.7 \%)$ & $<5(<5 \%)$ & $63(8.1 \%)$ & $5.97 \%$ & \\
\hline Allergic reaction & $27(3.1 \%)$ & $<5(1.1 \%)$ & $26(3.4 \%)$ & $3.70 \%$ & \\
\hline Cardiovascular & $31(3.6 \%)$ & $<5(1.1 \%)$ & $30(3.9 \%)$ & $3.23 \%$ & \\
\hline Altered mental status & $12(1.4 \%)$ & $5(5.3 \%)$ & $7(0.9 \%)$ & $41.67 \%$ & \\
\hline Minor trauma & $406(46.6 \%)$ & $39(41.1 \%)$ & $367(47.2 \%)$ & $9.61 \%$ & \\
\hline Major trauma & $8(0.9 \%)$ & $4(4.2 \%)$ & $4(0.5 \%)$ & $50.00 \%$ & \\
\hline Environmental & $2(0.2 \%)$ & $0(0 \%)$ & $2(0.3 \%)$ & $0.00 \%$ & \\
\hline Obstetrics & $1(0.1 \%)$ & $0(0 \%)$ & $1(0.1 \%)$ & $0.00 \%$ & \\
\hline Diabetic problem & $2(0.2 \%)$ & $1(1.1 \%)$ & $1(0.1 \%)$ & $50.00 \%$ & \\
\hline Wellness check & $74(8.5 \%)$ & $3(3.2 \%)$ & $71(9.1 \%)$ & $4.05 \%$ & \\
\hline Other & $44(5.1 \%)$ & $4(4.2 \%)$ & $40(5.2 \%)$ & $9.09 \%$ & \\
\hline Respiratory & $44(5.1 \%)$ & $5(5.3 \%)$ & $39(5 \%)$ & $11.36 \%$ & \\
\hline Overdose/poisoning & $41(4.7 \%)$ & $10(10.5 \%)$ & $31(4 \%)$ & $24.39 \%$ & \\
\hline Psychological & $27(3.1 \%)$ & $4(4.2 \%)$ & $23(3 \%)$ & $14.81 \%$ & \\
\hline Neuro & $78(8.9 \%)$ & $14(14.7 \%)$ & $64(8.2 \%)$ & $17.95 \%$ & \\
\hline Transfer & $8(0.9 \%)$ & $0(0 \%)$ & $8(1 \%)$ & $0.00 \%$ & \\
\hline
\end{tabular}

TABLE 3: Proportion of secondary transfers by chief complaint 


\section{Discussion}

This retrospective, observational cohort study found that increasing acuity and certain chief complaints had more frequent secondary transfer to pediatric specialty care within 24 hours.

"Major trauma" had a 50.0\% proportion of secondary transfer, which was tied for highest. Decreased mortality in children brought directly to pediatric trauma centres has been demonstrated previously, and many EMS systems, including the one serving the study area, have destination guidelines for pediatric trauma [3]. Although additional education about pediatric field triage guidelines may help providers to appropriately transport major trauma patients directly to the pediatric trauma centre, the paramedics who transported these trauma patients may have had justification for deviating from destination policy, such as requiring assistance with an airway.

"Diabetic complaint" was found to have a secondary transfer proportion of $50.0 \%$ although there were only two patients with this chief complaint transported to community hospitals during the study period. It is unclear if the incidence of pediatric diabetic issues is low or if these patients were being informally directed towards the pediatric centre. Knowlton et al. previously demonstrated that chronic medical conditions, such as diabetes, are linked to increased ambulance usage, but there are no published studies examining whether these patients benefit from specialized care [4].

"Altered mental status" had the third-highest transfer proportion (41.7\%). A variety of infectious, neurologic, metabolic or toxicologic causes can lead to this presentation which entails significant cerebral dysfunction. Prehospital level of consciousness may be a meaningful although non-specific indicator of severity in pediatric medical illness.

Patients transported to community hospitals with a complaint of "overdose/poisoning" had a transfer proportion of $24.4 \%$. Epidemiologic studies have found that the majority of these presentations are intentional ingestions and intoxication $[5,6]$. These patients may require mental health assessment which is only available at the pediatric specialty centre in our community. A recent study of adolescents visiting EDs in the United States found a sixfold increase in the odds of transfer or admission for those presenting with mental health complaints. Further study may be of benefit since pediatric patients with mental health and substance abuse have a higher proportion of repeat EMS usage [4].

\section{Conclusions}

In our retrospective study, pediatric patients transported by EMS to a community hospital with complaints of major trauma, diabetic complaint, altered mental status or overdose/poisoning were subsequently transferred to tertiary pediatric care more frequently than other chief complaints. Higher acuity patients, based on the CTAS score, also had a significantly higher percentage of secondary transfer to specialized pediatric care. These findings may help derive a decision tool to identify which pediatric patients would benefit from bypassing closer EDs in favour of transport directly to pediatric specialty care.

\section{Additional Information \\ Disclosures}

Human subjects: Consent was obtained by all participants in this study. Nova Scotia Health Authority Research Ethics Board issued approval 1021022. This research project was approved by the Nova Scotia Health Authority Research Ethics Board. Animal subjects: All authors have confirmed that this study did not involve animal subjects or tissue. Conflicts of interest: In compliance with the ICMJE uniform disclosure form, all authors declare the following: Payment/services info: This research was supported by the Dalhousie University Department of Emergency Medicine Division of EMS, Research Performance Bursary. Financial relationships: All authors have declared that they have no financial relationships at present or within the previous three years with any organizations that might have an interest in the submitted work. Other relationships: All authors have declared that there are no other relationships or activities that could appear to have influenced the submitted work.

\section{References}

1. Le May MR, Davies RF, Dionne R, et al.: Comparison of early mortality of paramedic-diagnosed ST-segment elevation myocardial infarction with immediate transport to a designated primary percutaneous coronary intervention center to that of similar patients transported to the nearest hospital. Am J Cardiol. 2006, 98:1329-1333. 10.1016/j.amjcard.2006.06.019

2. Browne LR, Shah MI, Studnek JR, et al.: 2015 Pediatric research priorities in prehospital care. Prehosp Emerg Care. 2016, 20:311-316. 10.3109/10903127.2015.1102997

3. Sathya C, Alali AS, Wales PW, et al.: Mortality among injured children treated at different trauma center types. JAMA Surg. 2015, 150:874-881. 10.1001/jamasurg.2015.1121

4. Knowlton AR, Weir B, Fields J, et al.: Pediatric use of emergency medical services: the role of chronic illnesses and behavioral health problems. Prehosp Emerg Care. 2016, 20:362-368.

10.3109/10903127.2015.1115928 


\section{Cureus}

5. Tyrell EG, Orton E, Tata LJ: Changes in poisonings among adolescents in the UK between 1992 and 2012: a population based cohort study. Inj Prev. 2016, 22:400-406. 10.1136/injuryprev-2015-041901

6. Cheng TL, Wright JL, Pearson-Fields AS, Brenner RA: DC Child/Adolescent injury research network. The spectrum of intoxication and poisonings among adolescents: surveillance in an urban population. Inj Prev. 2006, 12:129-132. 10.1136/ip.2005.010710 